\title{
Dystrophia myotonica type 1 presenting with dysarthria: A case report and literature review
}

\author{
CHUNRONG LI $^{*}$, XIAOLING ZHANG ${ }^{1 *}$, CHUNKUI ZHOU $^{1 *}$, LIJUN ZHU $^{2}$, \\ KANGDING LIU ${ }^{1}$ and SHAOKUAN FANG ${ }^{1}$ \\ ${ }^{1}$ Department of Neurology, Neuroscience Centre, The First Teaching Hospital of Jilin University, Changchun, Jilin 130012; \\ ${ }^{2}$ Department of Neurology, The Third Teaching Hospital of Jilin University, Changchun, Jilin 130021, P.R. China
}

Received October 13,2015; Accepted April 13, 2017

DOI: $10.3892 / \mathrm{etm} .2017 .4579$

\begin{abstract}
Dystrophia myotonica (DM) type 1 is an autosomal dominant disorder, caused by a trinucleotide CTG repeat expansion in the 3' untranslated region of the dystrophia myotonica protein kinase (DMPK) gene (chromosome 19q13.3). The disorder affects different organ systems, including the skeletal muscles, ocular lens, lungs, heart and gastrointestinal tract, as well as the endocrine and central nervous systems. The skeletal muscles are most frequently involved, whereby the disorder manifests as myotonia, muscle weakness and amyotrophy. However, DM type 1 presenting with dysarthria is rare. The current study presents a case of a 28-year-old male with DM type 1 presenting with dysarthria and associated multifocal hyperintense lesions in the white matter. Although electromyogram measurements identified myotonic discharges in all extremities, a muscle biopsy failed to detect the characteristic pathological features of DM type 1. A lack of a positive family history for DM type 1 also obscured diagnosis. However, genetic analysis detected a single allele in the P12 segment of the DMPK gene that included a CTG expansion of 13 repeats and a three-base gradient fragment in the P134 segment that included a CTG expansion of $>600$ repeats. According to the characteristics of dysarthria, multifocal hyperintense lesions in the white matter, electromyogram measurement results and genetic testing results, a diagnosis of DM type 1 was confirmed.
\end{abstract}

Correspondence to: Professor Shaokuan Fang, Department of Neurology, Neuroscience Centre, The First Teaching Hospital of Jilin University, 71 Xinmin Street, Changchun, Jilin 130012, P.R. China

E-mail: fang20063536@sina.com

${ }^{*}$ Contributed equally

Key words: dystrophia myotonica, dysarthria, case report, genetic analysis

\section{Introduction}

Dystrophiamyotonica (DM) is a systemic autosomal dominant disorder and follows a progressive, chronic course $(1,2)$. Incidence rates of DM range from 1 in 500 to 3 per 100,000, depending on the population (3). DM affects the skeletal muscles, ocular lens, lungs, heart and gastrointestinal tract, as well as the endocrine and central nervous systems $(1,2,4)$. The clinical manifestations of DM vary, however typical symptoms include myotonia, weakness and atrophy of the skeletal muscles $(5,6)$. Poor sleep quality, fatigue and excessive daytime sleepiness have an important effect on the quality of life of DM patients. Mathieu et al (7) proposed that DM mortality rate was 7.3 times higher than that of the normal population, and the average age of death was 53 years old in one 10 -year follow-up study. Seventy percent of cases of mortality in DM are associated with cardiorespiratory disorders and there is solid evidence that timely intervention and active monitoring significantly reduces morbidity and mortality, although there is no curative therapy (8).

DM belongs to the RNA-dominant disorders of the family and are autosomal dominant neuromuscular diseases caused by microsatellite expansions (9). Based on the mutations that occur within the DM protein kinase $(D M P K)$ gene, DM is categorized as two distinct types: Types 1 and 2 (5). DM type 1 is caused by expansion of CTG triplet repeat in the 3 ' untranslated region of the DMPK gene on chromosome 19q13.3 (5). DM type 2 is caused by the progressive expansion of a CCTG tetramer repeat in ZNF9. To date, only a small number of cases of white matter lesions in patients with DM1 have been reported. The current study presents the case of a 28-year-old male diagnosed with DM type 1 , who also presented with dysarthria and associated multifocal hyperintense lesions in the white matter. Diagnosis of DM type 1 was confirmed by genetic analysis.

\section{Case report}

A 28-year-old man presented with a 6-year history of progressive dysarthria and 2-year history of bilateral hand weakness. The patient was admitted to the Department of Neurology in the First Teaching Hospital of Jilin University (Changchun, China) in December 2014. On admission, the patient underwent 
physical examination that revealed exiguous hair, dysarthria, limited movement of the eyes, facial muscle weakness, glossal amyotrophy, bilateral sternocleidomastoid atrophy and left major-thenar amyotrophy. Muscle strength in the hands and muscle tone of all four limbs was decreased and a lack of tendon reflexes was observed in the extremities. Furthermore, bilateral mammary gland hyperplasia was detected. Magnetic resonance imaging (MRI) scans of the brain identified multifocal hyperintense lesions in the white matter of the bilateral parietal and frontal lobes, which were suggestive of demyelination (Fig. 1). The patient complained of a 4-year history of intermittent diarrhea. The patient denied any relevant family history of DM-associated pathologies. Written informed consent was provided by the patient prior to the current study.

Laboratory tests identified elevated levels of progesterone $[0.30 \mathrm{nmol} / 1$ (normal range, $0.138-2.671 \mathrm{nmol} / \mathrm{l})]$ and luteinizing hormone [9.518 $\mathrm{mIU} / \mathrm{ml}$ (normal range 1.24-8.62 $\mathrm{mIU} / \mathrm{ml}$ )], as well as reduced levels of vitamin B12 [107.59 pmol/1 (normal range 133-675 pmol/l)]. Creatinase levels, blood routine examination, conventional coagulation parameters, liver and kidney function, pituitary endocrine examination, hypersensitive C-reactive protein level, cortisol urine levels and glycosylated hemoglobin levels were all normal. An electrocardiogram detected first-degree atrioventricular block and ST segment elevation in leads II, III and avf. Electromyography (EMG) identified myotonic discharges in all extremities.

Biopsy of the right bicep muscle was performed according to routine surgical aseptic operation under local infiltration anesthetic. A dose of 5-20 ml of $1 \%$ lidocaine (Shanghai Zhaohui Pharmaceutical Co., Ltd.) was used (10). Fresh muscle specimens were placed in tragacanth on a piece of cork and inverted into liquid nitrogen cooled isopentane, shaken gently and removed after 20-30 sec. The muscle was then placed in a cryostat and cut into $6-\mu \mathrm{m}$ sections at $-25^{\circ} \mathrm{C}$. In general, based on the differential expression of isoforms of myosin heavy chains, skeletal muscle consists of four types of muscle fiber: Types I, IIA, IIB and IIX (11). Type I is a slow aerobic metabolism muscle fiber, which contains relatively more mitochondria and cytochromes and has low glycogen content (11). Subsequent hematoxylin stainig at $60^{\circ} \mathrm{C}$ for $30-60 \mathrm{sec}$ and eosin staining at room temperature for 1-3 min identified muscle fibers of variable sizes (primarily type I) and some atrophic fibers with an angular shape, observed by electron microscopy (Fig. 2). In addition, necrotic and regenerated fibers were identified, however, there was no evidence of sarcoplasmic reticulum or inflammatory cell infiltration. Enzymatic activity of nicotinamide adenine dinucleotide (NADH) and succinic dehydrogenase (SDH) was assayed by placing the slides in NADH and SDH incubating solution, containing NADH or SDH as a substrate and nitroblue tetrazolium for visualization of reaction for $1 \mathrm{~h}$ at $37^{\circ} \mathrm{C}$. NADH staining exhibited small type I fibers and reduced activity of NADH, observed by electron microscopy (Fig. 3).

Glutamyltranspeptidase staining was also performed. Fresh muscle specimens were placed in liquid nitrogen cooled isopentane to be frozen. Muscle tissue specimens were then placed at room temperature $\left(25^{\circ} \mathrm{C}\right)$, relative humidity $70 \%$. The dried frozen sections $(6 \mu \mathrm{m})$ were stained with hematoxylin for 10-20 $\mathrm{min}$, then rinsed with tap water for $5 \mathrm{~min}$ and dried with filter paper. The sections were then placed into a vat dye of Gomori trichrome stain for $10 \mathrm{~min}$ at room temperature $\left(25^{\circ} \mathrm{C}\right)$. The sections were differentiated using $0.2 \%$ acetic acid for $2 \mathrm{~min}$ at room temperature $\left(25^{\circ} \mathrm{C}\right)$. Sections were dehydrated in ascending alcohol solutions. The sections were cleared with xylene and mounted. The results did not reveal ragged red fibers by electron microscopy. In addition, no abnormalities were observed following staining with cytochrome oxidase, Periodic acid-Schiff or Oil red O (staining protocol as described for Gomori trichome stain).

The patient's genetic testing was performed using the fluorescent dye primer polymerase chain reaction (PCR) technique combined with the triple repeat primed PCR (TP-PCR) technique (12). Normal chromosome 19q13 was amplified by routine PCR. PCR products were detected by capillary electrophoresis. Abnormal chromosome 19q13 containing a mutation in the $D M P K$ gene was amplified into a series of bands on the gel by TP-PCR, due to presence of the PCR primer sequence within the mutant CTG repeat region (Fig. 4). Genetic analysis detected a single allele in the P12 segment of the DMPK gene that included a CTG expansion of 13 repeats (Fig. 5), as well as a three-base gradient fragment in the P134 segment including a CTG expansion of $>600$ repeats $(0-50$ repeats; Fig. 6$)$. The results of the genetic analysis were consistent with the pathological characteristics of DM type 1 . Thus, the patient was diagnosed with DM type 1 and orally treated with vitamin B1 $10 \mathrm{mg}$, vitamin B12 $500 \mu \mathrm{g}$ and folic acid $5 \mathrm{mg}$ three times a day. The patient was discharged after 4 days of hospitalization. Over a 5-month follow-up period, the patient experienced a marked improvement in muscular function and was able to perform daily activities.

\section{Discussion}

DM type 1 is one of the most prevalent hereditary neuromuscular disorders and follows a pattern of autosomal dominant inheritance. It typically affects skeletal and smooth muscle, as well as the endocrine and central nervous systems $(5,6,13)$. The disorder is characterized by progressive myopathy, myotonia and the involvement of multiple organs. Although the pathogenesis of DM type 1 is not fully understood, it is associated with a trinucleotide CTG repeat expansion in the 3'untranslated region of the $D M P K$ gene (chromosome 19q13) $(13,14)$.

Previous studies have focused on determining the pathogenetic mechanisms underlying the effects of the trinucleotide CTG repeat expansion on multi-systemic dysfunction $(15,16)$. The CTG triplet repeat expansion in the DMPK gene may cause nuclear localization of mutant mRNA. The mutant mRNA may then form RNA foci and sequestration of interacting RNA-binding proteins (15) Toxic repeat RNA sequences may potentially alter the regular expression of genes and the splicing process and may induce the abnormal expression of neuromuscular proteins, resulting in systemic manifestations including myotonia, muscle wasting, weakness and histopathology, cardiac conduction defects, cataracts and insulin resistance $(17,18)$.

DM type 1 is categorized into four distinct clinical forms: Adult-onset, congenital, childhood-onset and late-onset oligosymptomatic (19). The prognosis of DM type 1 is associated with the age of onset (20). Patients with childhood-onset DM typically experience poorer outcomes and have higher mortality 

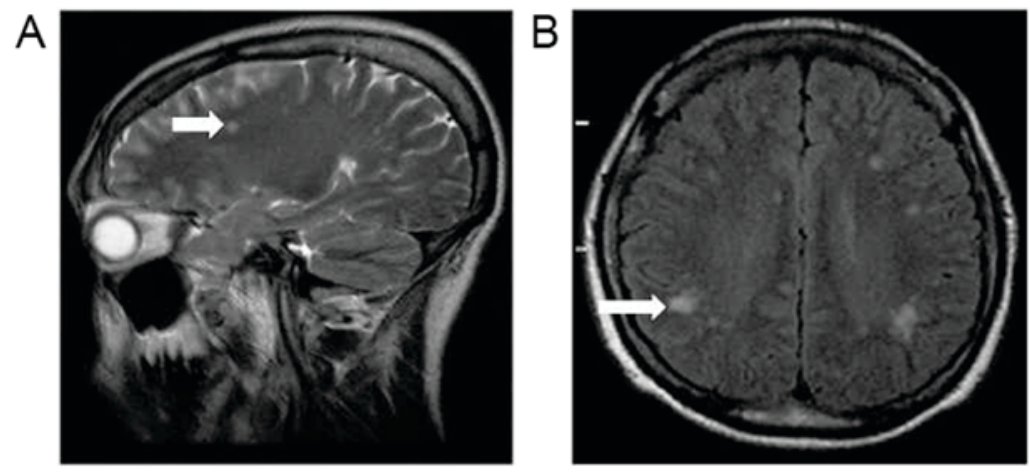

Figure 1. MRI scan of the patient. (A) T2-weighted image and (B) fluid attenuated inversion recovery MRI scan of the brain exhibiting bilateral, multifocal hyperintense lesions (white arrows) in the white-matter of the frontal (left) and parietal (right) lobes. MRI, magnetic resonance imaging.

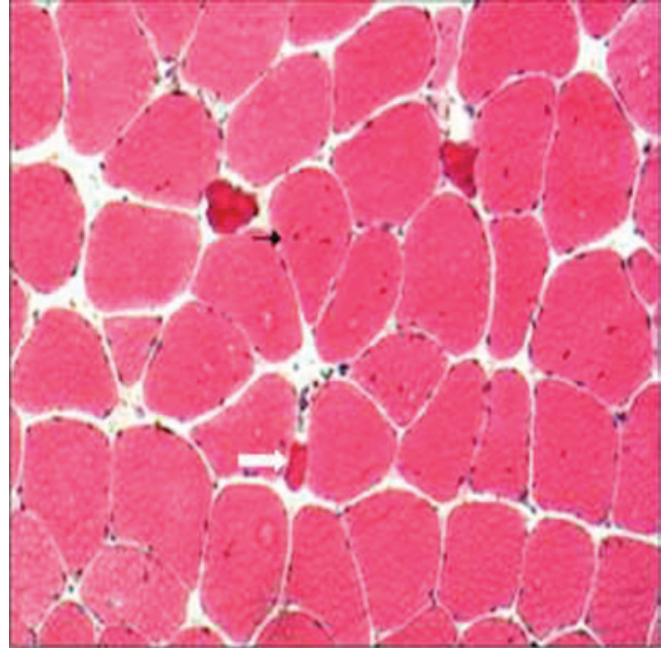

Figure 2. Image of stained right bicep tissue exhibiting muscular fibers of variable sizes and some atrophic fibers with an angular shape (white arrow). Larger internal nuclei of necrotic and regenerated fibers are also visible (indicated by the black arrow). Fibers lack sarcoplasmic reticulum or inflammatory cell infiltration. Magnification, x400. Hematoxylin and eosin staining.

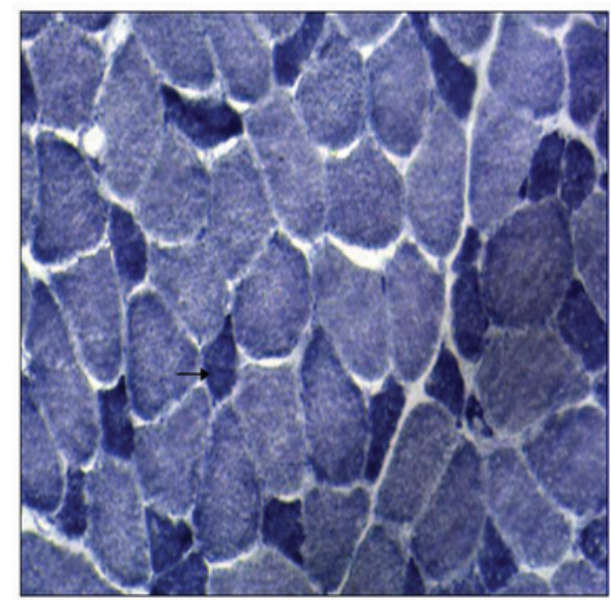

Figure 3. Dihydronicotinamide-adenine dinucleotide staining of the right bicep tissue. Image of stained right bicep tissue exhibiting small type I fibers (black arrow). Magnification, x400.

rates, whereas patients with adult-onset DM generally exhibit a more favorable prognosis (20). Adult-onset DM type 1, as

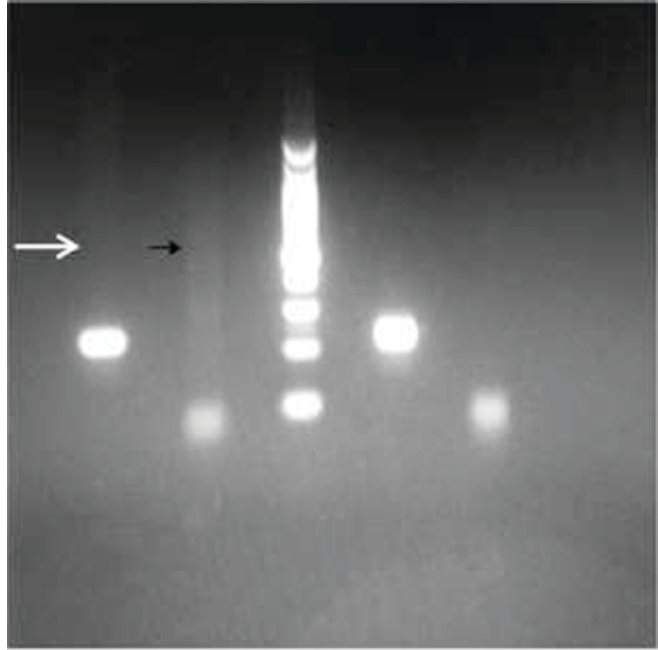

Figure 4. Electropherogram detection of PCR products. Normal chromosome 19q13 was amplified by routine PCR (D1: the products of PCR, white arrow). Abnormal chromosome 19q13 containing a mutation in the dystrophia myotonica protein kinase gene was amplified into a series of bands on the gel by tri-primer-PCR (D2: the products of TP-PCR, black arrow), due to presence of the PCR primer sequence within the mutant CTG repeat region. PCR, polymerase chain reaction.

documented in the present report, is the most prevalent form of the disorder (21). The primary clinical manifestations of DM type 1 are myotonia, muscle weakness and amyotrophy of the skeletal muscles. Myotonia is the most frequent symptom and typically manifests as difficulty in actively relaxing the thumb and/or fingers following contraction and may cause hypoventilation in some cases, due to stiffness of the respiratory or throat muscles. Amyotrophy initially affects the hand and forearm muscles but typically progresses to involve the head and facial muscles, and myotonia may present concomitantly with amyotrophy or precede it by a few years (22). The involvement of multiple organ systems may also complicate the pathophysiology of DM type 1 (23). For example, peristalsis in the gastrointestinal tract may become abnormal and potentially lead to abnormal rectum peristalsis, spasmodic colic and delayed emptying of the gallbladder, which ultimately results in cholelithiasis (17). Furthermore, endocrinal involvement in DM type 1 may lead to alopecia, impaired glucose tolerance, genital hypoplasia, sexual dysfunction and menstrual disorders (24). Central nervous system abnormalities observed in 


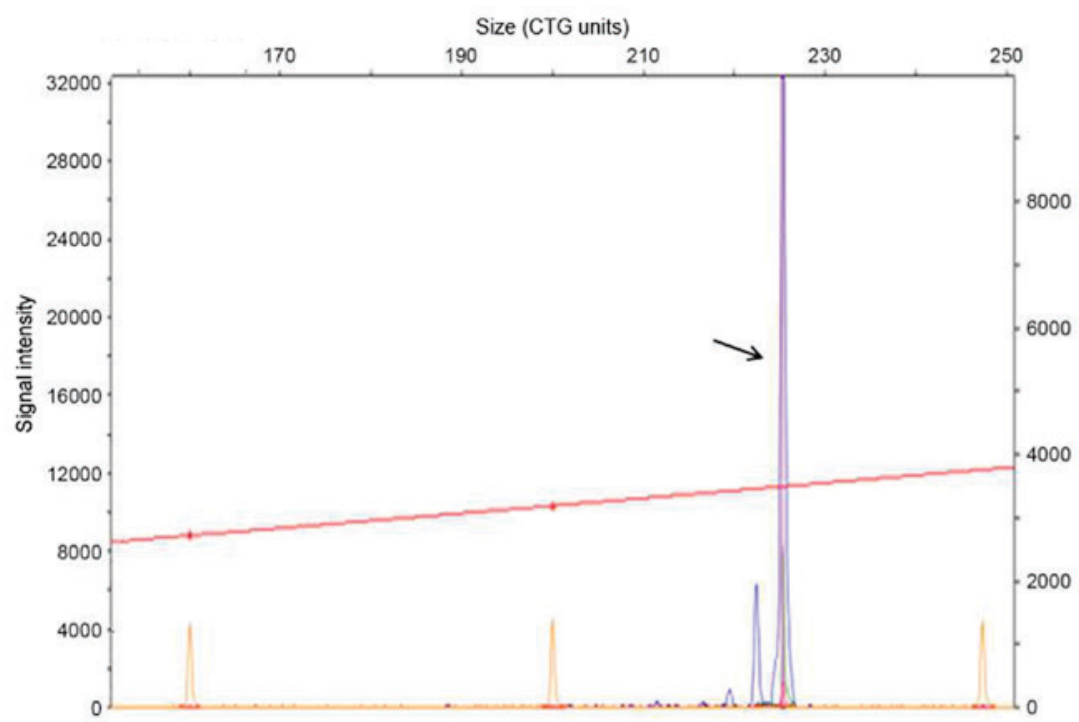

Figure 5. Genetic analysis of the P12 segment. Genetic testing identified a single allele in the P12 segment of the dystrophia myotonica protein kinase gene containing a CTG expansion of 13 repeats (black arrow).

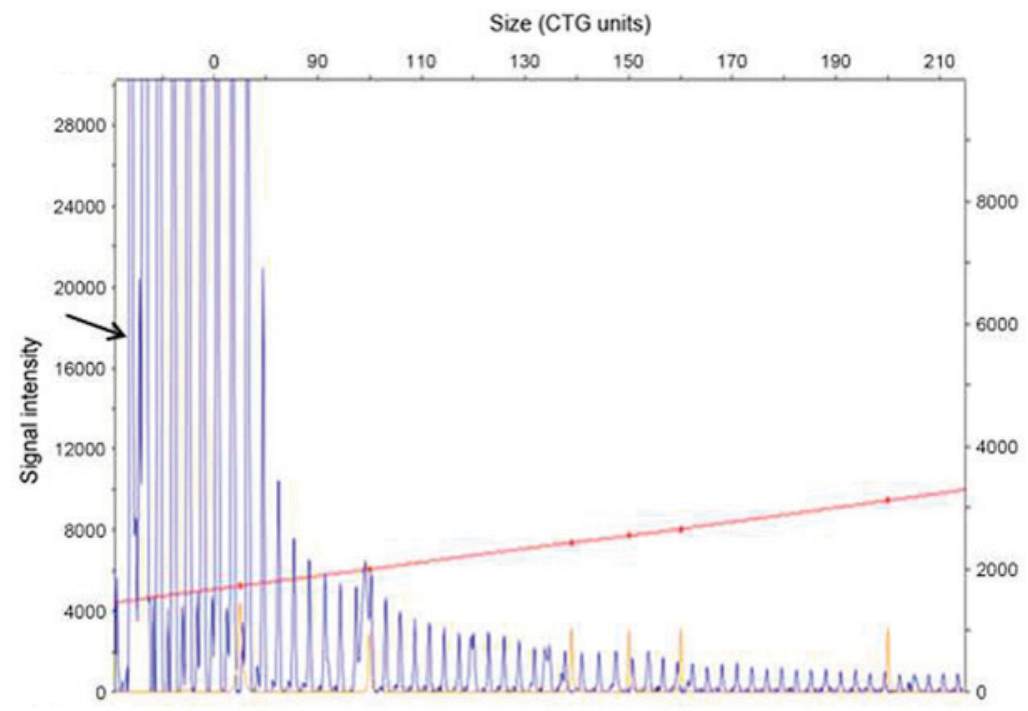

Figure 6. Genetic analysis of the P134 segment. Genetic testing identified a three-base gradient fragment in the P134 segment of the dystrophia myotonica protein kinase gene containing a CTG expansion of $>600$ repeats (black arrow).

patients with DM type 1 are structural and functional; patients typically present with hypophrenia and somnolence, and brain MRI scans indicate a thickened skull, narrowed sella, encephalatrophy and regional or diffuse white-matter changes (25). In addition, patients with DM type 1 are predisposed to develop cataracts and/or undergo retinal degenerative changes (21).

Diagnosis of DM type 1 is based on a combination of clinical manifestations, family history, EMG results, muscle histopathology and genetic testing. In the present case, the patient presented with chronic, progressive dysarthria, which initially obscured diagnosis. However a few years later, symptoms of myotonia, muscle weakness and amyotrophy became evident. No relevant family history of DM-associated pathologies was noted. A brain MRI scan identified multifocal hyperintense lesions in the white matter of the bilateral parietal and frontal lobes, suggestive of demyelination. As cases of DM type 1 with lesions in the white matter are rare (26), this was an unexpected result. It is not yet universally accepted that there is an association between white matter lesions and the severity of cognitive impairment (27). However, the present results suggest that conducting routine brain MRI scans in all patients with DM type 1 may be useful in diagnosing this disease, even in the absence of clear neurological manifestations.

It is important to differentiate DM type 1 from congenital myotonia (also known as myotonia congenital). There are two forms of congenital myotonia caused by mutations in the chloride voltage gated channel 1 gene located on chromosome $7 q 35$, which encodes the skeletal muscle chloride channel CIC-1 (28). The clinical presentation of congenital myotonia differs from that of DM type 1 in that it is typically accompanied by hypermyotrophy and systemic involvement other than that of the skeletal muscles is uncommon (29). 
In the present case report, the patient presented with multiple organ system involvement. Endocrinal involvement manifested as exiguous hair and bilateral mammary gland hyperplasia, whereas smooth muscle involvement was indicated by a 4-year history of intermittent diarrhea, potentially related to decreased gastrointestinal peristalsis and emptying. The ECG also identified a first-degree atrioventricular block and the brain MRI scan confirmed the presence of multifocal white matter lesions. The clinical symptoms in the present case were atypical. Although EMG identified myotonic discharges in all the extremities, a muscle biopsy failed to identify the characteristic pathologies of DM type 1. The absence of a family history of DM type 1 further inhibited the diagnosis. However, genetic analysis ultimately confirmed the diagnosis of DM type 1, indicating the value of genetic analysis as a diagnostic tool.

There are limited therapeutic options available for patients with DM type 1. Previous studies have indicated that membrane-depressant drugs, including phenytoin sodium and carbamazepine, may alleviate the symptoms of myotonia by promoting the activity of sodium pumps, leading to a reduction in intramembranous sodium concentration and an increase in resting membrane potential $(30,31)$. In addition, physical training may aid the maintenance of normal muscle functions (22). Cardiac arrhythmia is a primary cause of mortality in patients with DM type 1 and thus requires stringent monitoring during treatment. Cataracts associated with DM type 1 may be treated using conventional surgical strategies. In patients that exhibit endocrinal involvement, lifestyle changes, such as diet and exercise (8), are generally adequate to relieve symptoms. Recent progress in the understanding of the underlying molecular mechanisms involved in myotonic dystrophy have generated new approaches for DM type 1. Thus, future therapeutic strategies may employ gene therapy to treat genetic disorders such as DM.

\section{References}

1. Glaser AM, Johnston JH, Gleason WA and Rhoads JM: Myotonic dystrophy as a cause of colonicpseudoobstruction: Not just another constipated child. Clin Case Rep 3: 424-426, 2015.

2. Finsterer J, Karpatova A, Rauschka H, Loewe-Grgurin M, Frank M and Gencik M: Myotonic dystrophy 2 manifesting with non-alcoholic and non-hepatitic liver cirrhosis. Acta Clin Belg 70: 432-435, 2015.

3. Omond KJ and Byard RW: Forensic considerations in cases of myotonic dystrophy at autopsy. J Forensic Sci: Feb 7, 2017 (Epub ahead of print).

4. Tschuppert S and Gerding H: Myotonic dystrophy with reticular maculopathy as first ocular symptom. Klin Monbl Augenheilkd 232: 568-569, 2015.

5. Finsterer J and Rudnik-Schöneborn S: Myotonic dystrophies: Clinical presentation, pathogenesis, diagnostics and therapy. Fortschr Neurol Psychiatr 83: 9-17, 2015 (In German).

6. Thornton CA: Myotonic dystrophy. Neurol Clin 32: 705-719, viii, 2014.

7. Mathieu J, Allard P, Potvin L, Prévost C and Bégin P: A 10-year study of mortality in a cohort of patients with myotonic dystrophy. Neurology 52: 1658-1662, 1999.

8. Smith CA and Gutmann L: Myotonic dystrophy type 1 management and therapeutics. Curr Treat Options Neurol 18: 52, 2016.

9. Arandel L, Polay Espinoza M, Matloka M, Bazinet A, De Dea Diniz D, Naouar N, Rau F, Jollet A, Edom-Vovard F, Mamchaoui $\mathrm{K}$, et al: Immortalized human myotonic dystrophy muscle cell lines to assess therapeutic compounds. Dis Model Mech 10: 487-497, 2017.

10. Joyce NC, Oskarsson B and Jin LW: Muscle biopsy evaluation in neuromuscular disorders. Phys Med Rehabil Clin N Am 23: 609-631, 2012.
11. Zhang SH, Zhu L, Wu ZH, Zhang Y, Tang GQ, Jiang YZ, Li MZ, Bai L and Li XW: Effect of muscle-fiber type on glycogenin-1 gene expression and its relationship with the glycolytic potential and $\mathrm{pH}$ of pork. Genet Mol Res 12: 3383-3390, 2013.

12. Kamsteeg EJ, Kress W, Catalli C, Hertz JM, Witsch-Baumgartner M, Buckley MF, van Engelen BG, Schwartz $M$ and Scheffer H: Best practice guidelines and recommendations on the molecular diagnosis of myotonic dystrophy types 1 and 2. Eur J Hum Gene 20: 1203-1208, 2012.

13. Wissocque L, Brigadeau F, Richardson M, Boulé S, Kouakam C, Polge AS, Marquié C and Klug D: Impairment of global and regional longitudinal strains in patients with myotonic dystrophy type 1. Int J Cardiol 191: 46-47, 2015.

14. Gallais B, Montreuil M, Gargiulo M, Eymard B, Gagnon C and Laberge L: Prevalence and correlates of apathy in myotonic dystrophy type 1. BMC Neurol 15: 148, 2015.

15. Gladman JT, Mandal M, Srinivasan V and Mahadevan MS: Age of onset of RNA toxicity influences phenotypic severity: Evidence from an inducible mouse model of myotonic dystrophy (DM1). PLoS One 8: e72907, 2013.

16. Santoro M, Masciullo M, Silvestri G, Novelli G and Botta A: Myotonic dystrophy type 1: Role of CCG, CTC and CGG interruptions within DMPK alleles in the pathogenesis and molecular diagnosis. Clin Genet: Dec 19, 2016 (Epub ahead of print).

17. Lee JE, Bennett CF and Cooper TA: RNase H-mediated degradation of toxic RNA in myotonic dystrophy type 1. Proc Nat Acad Sci USA 109: 4221-4226, 2012.

18. Meola G and Cardani R: Myotonic dystrophies: An update on clinical aspects, genetic, pathology, and molecular pathomechanisms. Biochim Biophys Acta 1852: 594-606, 2015.

19. De Antonio M, Dogan C, Hamroun D, Mati M, Zerrouki S, Eymard B, Katsahian S and Bassez G; French Myotonic Dystrophy Clinical Network: Unravelling the myotonic dystrophy type 1 clinical spectrum: A systematic registry-based study with implications for disease classification. Rev Neurol (Paris) 172: 572-580, 2016.

20. Gagnon C, Kierkegaard M, Blackburn C, Chrestian N, Lavoie M, Bouchard MF and Mathieu J: Participation restriction in childhood phenotype of myotonic dystrophy type 1: A systematic retrospective chart review. Dev Med Child Neurol 59: 291-296, 2017.

21. Udd B and Krahe R: The myotonic dystrophies: Molecular, clinical, and therapeutic challenges. Lancet Neurol 11: 891-905, 2012.

22. Turner C and Hilton-Jones D: Myotonic dystrophy: Diagnosis, management and new therapies. Curr Opin Neurol 27: 599-606, 2014.

23. Groh WJ, Groh MR, Saha C, Kincaid JC, Simmons Z, Ciafaloni E, Pourmand R, Otten RF, Bhakta D, Nair GV, et al: Electrocardiographic abnormalities and sudden death in myotonic dystrophy type 1. N Engl J Med 358: 2688-2697, 2008.

24. Meola G and Sansone V: Cerebral involvement in myotonic dystrophies. Muscle Nerve 36: 294-306, 2007.

25. Kornblum C, Reul J, Kress W, Grothe C, Amanatidis N, Klockgether T and Schröder R: Cranial magnetic resonance imaging in genetically proven myotonic dystrophy type 1 and 2 . J Neurol 251: 710-714, 2004.

26. Liu L, Liu HM, Liu ZJ, Zhang LW, Gu WH and Wang RB: Myotonic dystrophy type 1 associated with white matter hyperintense lesions: Clinic, imaging, and genetic analysis. Chin Med J (Engl) 128: 1412-1414, 2015.

27. Minnerop M, Weber B, Schoene-Bake JC, Roeske S, Mirbach S, Anspach C, Schneider-Gold C, Betz RC, Helmstaedter C, Tittgemeyer M, et al: The brain in myotonic dystrophy 1 and 2: Evidence for a predominant white matter disease. Brain 134: 3530-3546, 2011.

28. Portaro S, Altamura C, Licata N, Camerino GM, Imbrici P, Musumeci O, Rodolico C, Conte Camerino D, Toscano A and Desaphy JF: Clinical, Molecular, and functional characterization of CLCN1 mutations in three families with recessive myotonia congenita. Neuromolecular Med 17: 285-296, 2015.

29. Lakraj AA, Miller G, Vortmeyer AO, Khokhar B, Nowak RJ and DiCapua DB: Novel mutations in the CLCN1 gene of myotonia congenita: 2 case reports. Yale J Biol Med 86: 101-106, 2013.

30. Kurihara T: New classification and treatment for myotonic disorders. Intern Med 44: 1027-1032, 2005.

31. Sechi GP, Traccis S, Durelli L, Monaco F and Mutani R: Carbamazepine versus diphenylhydantoin in the treatment of myotonia. Eur Neurol 22: 113-118, 1983. 